\title{
PRODUTIVIDADE DE MADEIRA DE LIQUIDAMBAR (Liquidambar styraciflua L.) DE DIFERENTES PROCEDÊNCIAS, EM QUEDAS DO IGUAÇU, PR'
}

\author{
Jarbas Yukio Shimizu², Ivana Helena Zamuner Spir ${ }^{3}$
}

\begin{abstract}
RESUMO - Foi instalado um teste combinado de procedências e progênies de liquidambar (Liquidambar styraciflua L.) em Quedas do Iguaçu, no sudoeste do Paraná, para verificar a produtividade de madeira e a variabilidade genética entre e dentro de procedências. O delineamento usado foi em blocos de famílias compactos, no espaçamento de $3 \mathrm{~m} \times 3 \mathrm{~m}$, com as progênies dispostas em parcelas lineares de cinco plantas. Aos 11 anos de idade, a produtividade média das procedências de maior crescimento foi maior que $40 \mathrm{~m}^{3} / \mathrm{ha}$. ano. As procedências de maior crescimento foram da América Central (Guatemala, Honduras e Nicarágua), originárias de latitudes inferiores a $15^{\circ} \mathrm{N}$. Nas procedências Gomez Farias, Las Lajas, Los Alpes e Monte Bello foram detectadas variações significativas entre suas progênies. Porém, somente as seleções entre as progênies oriundas de Los Alpes e Las Lajas proporcionariam oportunidades de melhoramento genético, tendo em vista as suas altas médias de produtividade. Não foram detectadas variações significativas em volume entre as progênies das procedências Tactic e Yucul. Porém, será oportuno incluí-las na população para melhoramento, dadas a elevada produtividade volumétrica e a oportunidade de se ampliar a base genética para seleções posteriores com base em outros caracteres, como a qualidade do fuste e da madeira.
\end{abstract}

Palavras-chave: Liquidambar styraciflua, progênie, seleção.

\section{WOOD PRODUCTIVITY IN SWEETGUM DERIVED FROM DIFFERENT PROVENANCES IN QUEDAS DO IGUAÇU, PR}

\begin{abstract}
A combined provenance/progeny trial with sweetgum (Liquidambar styraciflua L.) from Mexico and Central America was established in Quedas do Iguaçu, in the Southwest of the State of Paraná, in order to assess wood productivity and genetic variability. The trial was established on a sloping site and arranged in a compact family block design, with the progenies planted into 5-tree linear plots. At eleven years of age, the mean yield of the fastest growing provenances (all from Central America, from latitudes no higher than $15^{\circ} \mathrm{N}$ ) was higher than $40 \mathrm{~m}^{3} / \mathrm{ha}$.year. Significant variations were detected among progenies within Gomez Farias, Las Lajas, Los Alpes, and Monte Bello provenances. However, among these, only Los Alpes and Las Lajas provenances offer opportunities for genetic improvement of wood production through selection among their progenies since the mean productivity in the others were low. Although no significant variation was detected among progenies within Tactic and Yucul provenances, their inclusion in breeding populations is important, given their high mean productivity and the opportunity to increase population sizes for further selections based on other traits such as stem form and wood quality.
\end{abstract}

Key-words: Liquidambar styraciflua; progeny; selection.

\footnotetext{
${ }^{1}$ Recebido para publicação em 18.8.2003 e aceito para publicação em 10.8.2004.

${ }^{2}$ Embrapa Florestas, Caixa Postal 319.83411-000 Colombo, PR. E.mail: <jarbas@cnpf.embrapa.br>.

${ }^{3}$ Curso de Engenharia Florestal da UFPR. Curitiba, PR.
} 


\section{INTRODUÇÃO}

O liquidambar (Liquidambar styraciflua L.) é uma espécie arbórea, folhosa, de ampla distribuição natural no sul e sudeste dos Estados Unidos (estendendose desde Connecticut, em direção ao oeste, até o leste do Texas e desde as partes sul de Ohio, Indiana e Illinois para o sul até a região central da Flórida), estendendo-se, também, para o México e a América Central (KORMANIK, 1990). Uma importante característica desta espécie é a capacidade de se desenvolver em solos úmidos, onde a maioria das espécies arbóreas não teria condições de prosperar.

Em suas origens, a madeira do liquidambar é considerada de alta qualidade para usos gerais em construções, marcenaria e industrialização. Não apresenta grande oscilação de peso específico, mesmo variando a posição no tronco (CARPENTER e HOPKINS, 1966). Além disso, apresenta textura uniforme, dureza e densidade médias e facilidade de ser polida. Esses atributos, além da alta capacidade de adaptação e crescimento no Estado de São Paulo (GURGEL GARRIDO et al., 1997; SHIMIZU e SPIR, 1999), conferem à espécie uma posição relevante como alternativa para compor sistemas agroflorestais em pequenas e médias propriedades rurais ou em reflorestamentos intensivos, para produção de madeira para fins industriais.

Experimentos envolvendo procedências e progênies desta espécie têm demonstrado o seu potencial na região central do Estado de São Paulo, em solo arenoso com déficit hídrico em parte do ano (GOLFARI et al., 1978). Nesse ambiente, Shimizu e Spir (1999) constataram variações no crescimento em razão tanto da proce- dência das sementes quanto da posição no terreno. Porém, não há, ainda, registro do desempenho desta espécie na Região Sul, onde as condições climáticas e edáficas são distintas. Este estudo teve como objetivo avaliar a potencialidade da espécie para produção de madeira na região sudoeste do Paraná, onde o clima é frio, com ocorrência de geadas severas e solos argilosos sem déficit hídrico.

\section{MATERIAL E MÉTODOS}

Foi instalado um teste combinado de procedências e progênies de liquidambar, em Quedas do Iguaçu, no sudoeste do Paraná, na região Bioclimática 1 (EMBRAPA, 1986), que se caracteriza pela temperatura média anual entre 15 e $19^{\circ} \mathrm{C}$, precipitação média anual entre 1.250 e $2.500 \mathrm{~mm}$, com chuvas uniformemente distribuídas durante o ano, sem déficit hídrico. O local do plantio era ocupado por uma floresta secundária, e o preparo do solo consistiu somente de limpeza e coveamento. O material genético (semente), fornecido pelo Oxford Forestry Institute (HUGHES e McCARTER, 1984), foi coletado de matrizes em suas origens, gerado por polinização aberta, de oito procedências da América Central e México (Quadro 1).

O delineamento experimental usado foi o de blocos de famílias compactos, com as parcelas de progênies dispostas em linhas de cinco plantas, no espaçamento de $3 \mathrm{~m}$ x $3 \mathrm{~m}$ em sete repetições. Aos 11 anos, foram medidas as alturas e os diâmetros (DAP) para a estimação dos volumes individuais, aplicando-se o fator de forma 0,5 . No caso de árvores bifurcadas abaixo de 1,3 m de altura, mediu-se somente o tronco de maior diâmetro.

Quadro 1 - Características dos locais de origem das procedências de Liquidambar styraciflua testadas em Quedas do Iguaçu, PR Table 1 - Characteristics of Liquidambar styraciflua provenance tested in Quedas do Iguaçu, PR

\begin{tabular}{|c|c|c|c|c|c|}
\hline Procedências & $\begin{array}{l}\text { Latit. } \\
(\mathrm{N})\end{array}$ & $\begin{array}{l}\text { Long. } \\
\text { (W) }\end{array}$ & $\begin{array}{c}\mathrm{TMA}^{1} \\
\left({ }^{\circ} \mathrm{C}\right)\end{array}$ & $\begin{array}{l}\text { Altitude } \\
\text { (m) }\end{array}$ & $\begin{array}{l}\text { PMA }^{2} \\
(\mathrm{~mm})\end{array}$ \\
\hline Las Victorias, Sierra de las Minas, Guatemala & $15^{\circ} 12^{\prime}$ & $89^{\circ} 22^{\prime}$ & 20,0 & $800-1000$ & $1900-2100$ \\
\hline Los Alpes, Siguatepeque, Honduras & $14^{\circ} 33^{\prime}$ & $87^{\circ} 58^{\prime}$ & 18,0 & $1250-1350$ & $1400-1600$ \\
\hline Las Lajas, Comayagua, Honduras & $14^{\circ} 48^{\prime}$ & $87^{\circ} 34^{\prime}$ & 21,5 & $1100-1200$ & $1500-2000$ \\
\hline Yucul, Matagalpa, Nicaragua & $12^{\circ} 55^{\prime}$ & $85^{\circ} 48^{\prime}$ & 20,0 & $800-1100$ & $1400-1600$ \\
\hline Tactic, Coban, Guatemala & $15^{\circ} 19^{\prime}$ & $90^{\circ} 21$ & 17,5 & $1380-1420$ & 2079 \\
\hline Gomez Farias, Tamaulipas, México & $23^{\circ} 06^{\prime}$ & $99^{\circ} 10^{\prime}$ & 17,0 & $1100-1450$ & $1800-2400$ \\
\hline Lagunas de Monte Bello, Chiapas, México & $16^{\circ} 04^{\prime}$ & $91^{\circ} 44^{\prime}$ & 18,0 & $1460-1540$ & $1800-2000$ \\
\hline Zacualtipan, Hidalgo, México & $20^{\circ} 42^{\prime}$ & $98^{\circ} 37^{\prime}$ & 13,5 & $1800-1870$ & 2047 \\
\hline
\end{tabular}

FONTE: Hughes e McCarter (1984). ${ }^{1} \mathrm{TMA}=$ temperatura média anual. ${ }^{2} \mathrm{PMA}=$ precipitação média anual. 
Devido a mortalidades no campo, foram perdidas duas parcelas de progênies das procedências Tactic, Alpes e Yucul e uma da procedência Monte Bello. Assim, para viabilizar a análise de variância no modelo balanceado, os valores das parcelas perdidas foram estimados usando-se o procedimento descrito por Steel e Torrie (1980) e fazendo as devidas correções nos graus de liberdade dos erros experimentais.

Las Victorias e Las Lajas foram representadas por apenas uma e duas progênies, respectivamente, e seus dados não podem ser tomados como representativos do desempenho de suas procedências, porém esses valores foram incluídos para efeito comparativo. Da mesma forma, as procedências Zacualtipan e Tactic com cinco progênies e Los Alpes com quatro podem ser consideradas ainda insuficientemente representadas para se ter uma avaliação plenamente confiável dessas procedências. Isto porque, para a formação da população para melhoramento (área de produção de semente ou pomar de semente), serão incluídas árvores de maior valor individual; independentemente da procedência, todas as progênies serão avaliadas no conjunto.

\section{RESULTADOS E DISCUSSÃO}

A região de Quedas do Iguaçu mostrou-se alta- mente favorável ao desenvolvimento do liquidambar. Ao contrário do que foi observado na região central do Estado de São Paulo (SHIMIZU e SPIR, 1999), em Quedas do Iguaçu não foi detectado efeito dos blocos (Quadro 2). As razões prováveis para essa resposta poderiam ser as condições ambientais propícias ao pleno crescimento da espécie durante o ano todo, dada a aparente uniformidade do solo em toda a extensão do terreno e a ausência de déficit hídrico.

As variações entre as procedências e progênies testadas foram altamente significativas (Quadro 2). Somente nas procedências Gomez Farias, Las Lajas, Los Alpes e Monte Bello foram detectadas variações estatisticamente significativas entre as respectivas progênies. Os coeficientes de variação do erro experimental $(\mathrm{CV})$ da altura e do DAP mantiveram-se em níveis razoáveis, permitindo uma análise confiável. Os altos valores de CV verificados em volume foram decorrentes da composição dos erros nas variáveis altura e DAP, usados na sua estimação. Poder-se-ia considerar a não-detecção de variações significativas em volume entre progênies, nas procedências Zacualtipan, Tactic e Yucul, como decorrente do alto valor do CV. No entanto, isso é pouco provável, visto que não foram detectadas variações significativas em altura nem em DAP, nos quais os CV foram baixos. Consi-

Quadro 2 - Análises de variância de procedências e progênies de Liquidambar styraciflua aos 11 anos, em Quedas do Iguaçu, PR

Table 2 - Analysis of variance of 1-year old Liquidambar styraciflua provenance and progenies in Quedas do Iguaçu, PR

\begin{tabular}{|c|c|c|c|c|c|c|c|}
\hline \multirow[b]{2}{*}{ Fonte de Var. } & \multirow[b]{2}{*}{$\mathrm{GL}^{11}$} & \multicolumn{2}{|c|}{ Altura } & \multicolumn{2}{|c|}{ DAP } & \multicolumn{2}{|c|}{ VOLUME } \\
\hline & & $\mathrm{QM}^{12}$ & $\mathrm{CV}^{1 / 3}$ & QM & $\mathrm{CV}$ & QM & $\mathrm{CV}$ \\
\hline Blocos & 6 & 2,64313 & & $15,81215^{*}$ & & 0,01634 & \\
\hline Tratamentos & 41 & $7,10409 * *$ & 6,01 & $39,73397 * *$ & 11,66 & $0,07294 * *$ & 23,08 \\
\hline Entre procedências & 7 & $5,72895 * *$ & 4,27 & $40,81918 * *$ & 6,42 & $0,07850 * *$ & 12,69 \\
\hline Entre prog./Zacualtipan & 4 & 2,86116 & 6,70 & 6,94114 & 11,49 & 0,01326 & 27,35 \\
\hline Entre prog./Tactic & 4 & 1,53627 & 7,48 & 13,25025 & 11,12 & 0,01823 & 22,83 \\
\hline Entre prog./Gomez & 7 & $6,13726^{* *}$ & 6,14 & $27,89836^{* *}$ & 10,53 & $0,04179 * *$ & 23,11 \\
\hline Entre prog./Las Lajas & 1 & 0,12698 & 3,05 & $29,95931 * *$ & 4,46 & $0,00334 * *$ & 10,64 \\
\hline Entre prog./Alpes & 3 & 1,70016 & 5,57 & $41,14981 * *$ & 10,10 & $0,10508 * *$ & 21,88 \\
\hline Entre prog./Monte Bello & 8 & 4,63352 & 7,51 & $26,42488 * *$ & 11,48 & $0,03878 * *$ & 23,76 \\
\hline Entre prog./Yucul & 7 & 1,90603 & 6,01 & 13,16335 & 11,66 & 0,02319 & 23,08 \\
\hline RESÍDUO & 239 & 1,91744 & & 6,76460 & & 0,01017 & \\
\hline
\end{tabular}

TOTAL 286

$\underline{1}=$ graus de liberdade.

$\underline{12}=$ efeitos significativos com $99 \%(* *)$ e $95 \%$ de probabilidade $(*)$, pelo teste $\mathrm{F}$.

$\underline{\underline{B}}=$ coeficiente de variação do erro experimental, em $\%$. 
derando, ainda, a correlação normalmente alta entre o DAP e o volume, pode-se inferir que as variações entre as progênies, nessas procedências, não foram substanciais. Exclui-se dessa avaliação a procedência Las Victorias, visto estar representada por apenas uma progênie.

Tomando as médias de produção volumétrica por árvore de cada procedência, observou-se que, no geral, o material genético originário do estado de Chiapas (México) e mais ao norte, de latitudes maiores que $16^{\circ}$ $\mathrm{N}$ e de maiores altitudes, cresceu menos que o originário da América Central, de latitudes menores que $15^{\circ} \mathrm{N}$. As três procedências de menor incremento volumétrico foram originárias do México, e as demais de Honduras, Guatemala e Nicarágua. Fato semelhante foi observado em Agudos, SP (SHIMIZU e SPIR, 1999).

Tomando-se o desempenho somente das procedências da América Central, plantadas no espaçamento de $3 \mathrm{~m} \times 3 \mathrm{~m}$, e supondo uma mortalidade de até $20 \%$ ao longo dos anos, o incremento médio anual, até os 11 anos de idade, foi estimado em mais de $40 \mathrm{~m}^{3} /$ ha.ano. Essa produtividade foi equivalente à dos eucaliptos de rápido crescimento. Além disso, o liquidambar oferece as vantagens de ser apropriado para a região de ocorrência de geadas e de produzir madeira de alta qualidade, tanto para produção de celulose e papel quanto para a indústria de madeira serrada e laminada, mesmo em sítios com alta umidade no solo.

Para implementar um programa de melhoramento genético, sugere-se selecionar indivíduos não só das procedências Los Alpes e Las Lajas, que demonstraram alta produção volumétrica e alto potencial de resposta à seleção pelo volume, mas, também, indivíduos das procedências Yucul e Tactic, que, apesar de não terem apresentado variação entre progênies, exibiram alta produtividade média e poderão contribuir com a ampliação da base genética para seleções posteriores de caracteres adicionais, como qualidade do fuste e da madeira. Apesar das variações estatisticamente significativas observadas entre as progênies das procedências Gomez Farias e Monte Bello, esse material genético não ofereceu oportunidade para o melhoramento genético da produção de madeira de liquidambar em Quedas do Iguaçu, dada a baixa produtividade média que apresentou.

\section{CONCLUSÕES}

O liquidambar é uma espécie alternativa valiosa para plantios florestais no sudoeste do Paraná, com potencial de produtividade de madeira em torno de $40 \mathrm{~m}^{3} /$ ha.ano. Nessa região, o material genético originado da América Central, de latitudes inferiores a $15^{\circ} \mathrm{N}$, produz maiores volumes de madeira do que o de latitudes superiores. Portanto, sementes oriundas ou derivadas dessas fontes, que incluem Guatemala, Honduras e Nicarágua (não as do México), são as de maior potencial produtivo. Na procedência Los Alpes e Las Lajas, existe variação genética, que pode ser explorada visando ao aumento da produtividade de madeira por meio da seleção de matrizes.

Quadro 3 - Médias das procedências (em negrito) e amplitude de variação entre progênies de Liquidambar styraciflua aos 11 anos, em Quedas do Iguaçu, PR

Table 3 - Provenance means (in bold) and range of progeny means in 11-year old Liquidambar styraciflua in Quedas do Iguaçu, $P R$

\begin{tabular}{lccc}
\hline PROCEDENCIAS & ALT $(\mathrm{m})$ & DAP $(\mathrm{cm})$ & VOL $\left(\mathrm{m}^{3} /\right.$ árvore $)$ \\
\hline Las Victorias* & $\mathbf{2 0 , 9}$ & $\mathbf{2 8 , 0}$ & $\mathbf{0 , 6 5 0 1 6}$ \\
Los Alpes & $20,6-\mathbf{2 1 , \mathbf { 1 }}-21,8$ & $24,2-\mathbf{2 6 , 1}-29,4$ & $0,49278-\mathbf{0 , 5 8 3 1 0}-0,75350$ \\
Las Lajas & $21,6-\mathbf{2 1 , 7}-21,8$ & $23,6-\mathbf{2 5 , 0}-26,5$ & $0,47661-\mathbf{0 , 5 4 3 4 3}-0,61025$ \\
Yucul & $19,9-\mathbf{2 1 , 0}-21,6$ & $21,3-\mathbf{2 3 , \mathbf { 8 }}-25,1$ & $0,39114-\mathbf{0 , 4 7 7 8 5}-0,54284$ \\
Tactic & $19,8-\mathbf{2 0 , 4}-21,1$ & $21,4-\mathbf{2 3 , 3}-25,0$ & $0,37051-\mathbf{0 , 4 3 1 4 8}-0,49716$ \\
Gomez Farias & $18,1-\mathbf{1 9 , 4}-20,9$ & $19,9-\mathbf{2 2 , 1}-25,3$ & $0,31433-\mathbf{0 , 3 9 6 2 4}-0,52254$ \\
Monte Bello & $19,0-\mathbf{2 0 , 4}-21,7$ & $18,6-\mathbf{2 1 , 1}-24,2$ & $0,28293-\mathbf{0 , 3 7 2 9 2}-0,48618$ \\
Zacualtipan & $17,9-\mathbf{1 9 , 0}-19,6$ & $20,1-\mathbf{2 1 , 5}-22,8$ & $0,29606-\mathbf{0 , 3 6 1 1 7}-0,41315$ \\
\hline
\end{tabular}

* Procedência representada por uma só progênie. 


\section{AGRADECIMENTOS}

Os autores agradecem à empresa ARAUPEL, na pessoa do Eng. Florestal Saulo Barbieri, pela colaboração na instalação, manutenção e medição do experimento, tendo, assim, possibilitado a realização deste estudo.

\section{REFERÊNCIAS BIBLIOGRÁFICAS}

CARPENTER, B. E.; HOPKINS, W. C. Specific gravity values of sweetgum (Liquidambar styraciflua) topwood and bolewood. Forest Products Journal, v.6, n.7, p.30, 1966.

EMPRESA BRASILEIRA DE PESQUISA AGROPECUÁRIA - EMBRAPA. Zoneamento ecológico para plantios florestais no Estado do Paraná. Brasília: 1986. 89p.

GOLFARI, L.; CASER, R. L.; MOURA, V.P. G. Zoneamento ecológico esquemático para reflorestamento no Brasil. Belo Horizonte: 1978. 66p (PNUD/FAO/IBDF/BRA-45. Série Técnica, 1).
GURGEL GARRIDO, L. M. A. et al. Variabilidade genética de características silviculturais de Liquidambar styraciflua $\mathrm{L}$. em teste de origens em Paraguaçu Paulista - SP. Revista do

Instituto Florestal, São Paulo, v.9, n.2, p.125$132,1997$.

HUGHES, C. E.; McCARTER, P. S. Exploration and seed collection of Liquidambar styraciflua in Central America and Mexico. In: IUFRO Working Parties on Provenance and Genetic Improvement Strategies in Tropical Forest Trees. Mutare, Zimbabwe, 1984.

KORMANIK, P. P. Liquidambar styraciflua L. Sweetgum. In: SILVICS OF NORTH AMERICA. Hardwoods. Washington: USDA, 1990. v.2. p.400-405. (Agriculture Handbook, 654).

SHIMIZU, J. Y.; SPIR, I. H. Z. Avaliação de procedências e progênies de liquidambar da América Central, do México e dos Estados Unidos, em Agudos, Brazil. Colombo, Boletim de Pesquisa Florestal, n.39, p.93-108, 1999.

STEEL, R. G. D.; TORRIE, J. H. Principles and procedures of statistics: a biometrical approach. 2. ed. New York: McGraw-Hill, 1980. 633p. 